\title{
INTEGRAÇÃO DE SENSORIAMENTO REMOTO E MODELOS HIDROLÓGICOS PARA A ESTIMATIVA DA EVAPOTRANSPIRAÇÃO: UMA REVISÃO BIBLIOGRÁFICA.
}

\author{
Morris Scherer Warren*
}

Resumo: A hidrologia geográfica busca o entendimento do padrão espacial da água em sua fase terrestre. Para monitorar e simular a água terrestre, diferentes ferramentas tecnológicas têm sido utilizadas nos últimos anos, com especial destaque para o sensoriamento remoto e a modelagem hidrológica. O presente artigo traz uma revisão acerca da utilização do sensoriamento remoto e da modelagem hidrológica no entendimento da evapotranspiração, uma variável-chave na análise geográfica. O levantamento realizado mostra o benefício de se utilizar estimativas remotas de evapotranspiração para diminuir a incerteza das simulações realizadas com modelos hidrológicos, bem como da possibilidade de aprimoramento dos procedimentos de assimilação.

Palavras-chave: hidrologia, geoprocessamento, análise espacial.

\section{INTEGRATION OF REMOTE SENSING AND HYDROLOGICAL MODELS FOR THE ESTIMATION OF EVAPOTRANSPIRATION: A REVIEW}

\begin{abstract}
The aim of Geographical hydrology is the elucidation of the spatial patterns of water in the terrestrial phase. Different technological tools have been used in recent years to monitor and simulate terrestrial water dynamics and distribution, with emphasis on remote sensing and hydrological modelling techniques. This article presents a review on the use of these technological tools in the study of evapotranspiration, a key variable in geographical analysis. The results points to the benefits of using estimations of remotely sensed evapotranspiration to reduce uncertainty in hydrological modelling simulations, and the possibility of improving assimilation techniques.
\end{abstract}

Keywords: hydrology, geomatics, spatial analysis.

\section{Introdução}

Em seus clássicos livros de hidrologia geográfica, Chorley (1971) e Knapp (1979) lançam as bases de uma hidrologia científica, em detrimento de uma perspectiva voltada exclusivamente para a engenharia. A hidrologia geográfica busca o entendimento dos processos que alteram os fluxos de água ao longo do ciclo hidrológico terrestre, sua distribuição espacial na bacia hidrográfica e a influência de fatores antrópicos e naturais.

Dessa época em diante houve significativa evolução das chamadas "geotecnologias", ou seja, ferramentas tecnológicas voltadas para a aquisição, armazenamento e análise de informações espaciais, abrindo novas possibilidades para a hidrologia geográfica. Em particular, o sensoriamento remoto e modelos hidrológicos têm sido utilizados para o monitoramento e a simulação da água em sua fase terrestre, bem como para a análise integrada entre recursos hídricos, uso da terra e mudanças climáticas.

O presente artigo traz uma revisão acerca das possibilidades e limitações atuais do uso de sensoriamento remoto na hidrologia geográfica. O foco da revisão será na evapotranspiração, pois essa variável liga

\footnotetext{
* Formado em Geografia pela Universidade Federal de Santa Catariana. Mestrado em Sensiriamento Remoto pelo Inpe. Doutor em Tecnologia Ambiental e Recursos Hídricos. Trabalha desde 2004 na Agência Nacional de Águas. E-mail: morris@ana.gov.br.
} 
o uso da terra e da água com a disponibilidade hídrica da bacia hidrográfica, sendo de particular interesse na análise geográfica. A possibilidade de integração de modelos hidrológicos e sensoriamento remoto no entendimento da dinâmica espacial da evapotranspiração também será abordada.

\section{Modelos hidrológicos}

Modelos são abstrações da realidade que visam representar simplificadamente o comportamento de um sistema. O melhor modelo é aquele que atinge o maior realismo com a menor complexidade de representação, medido objetivamente como a concordância entre a saída de um modelo e uma observação do mundo real (MULLIGAN; WAINWRIGHT, 2004).

Em hidrologia, modelos visam simular os fluxos e armazenamentos da água e sua qualidade no contexto da bacia hidrográfica. Essa definição ampla inclui a estimativa de vazões e níveis dos rios, inventários dos recursos hídricos, análise dos impactos das alterações do uso da terra sobre os recursos hídricos, propagação de sedimentos e poluição, simulação dos efeitos de mudanças climáticas, impactos sobre a ecologia aquática, entre outros (TUCCI, 2005).

Refsgaard (1996) apresenta uma classificação de modelos de simulação hidrológica (Figura 1). Em um primeiro nível é apresentada a distinção entre modelos determinísticos e estocásticos.

Modelos determinísticos são classificados em três categorias: empíricos (caixa-preta); conceituais-concentrados (caixa-cinza) e modelos distribuídos de bases físicas (caixa-branca). Modelos caixa-preta são empíricos, ou seja, a precipitação é transformada em vazão pela utilização de técnicas estatísticas, sem considerar explicitamente os processos físicos da bacia. Modelos conceituais procuram representar de forma concentrada (valores médios) os fluxos e armazenamentos em diferentes elementos da bacia. Modelos distribuídos de bases físicas calculam diretamente os fluxos de água e/ou energia por equações diferenciais parciais, como a equação de Saint Venant para escoamento em vertentes e canais, a equação de Richard para fluxo não saturado no solo e a equação de Boussineq para fluxo de água subterrânea (DHI, 2008). São modelos que permitem uma associação direta com as características físicas da bacia, seja vegetação, solo ou geologia.

Modelos estocásticos são derivados da análise de séries temporais, na qual uma sequência de eventos é tratada como dependente do tempo. O modelo estocástico pode ser usado para a extrapolação temporal da série de vazões, usando para isto as mesmas propriedades estatísticas da série histórica observada.

Por fim, modelos estocásticos-determinísticos são combinações dessas duas classes de modelos, tendo como objetivo a consideração de incertezas no processo de modelagem determinística. A técnica de Monte Carlo, por exemplo, pode ser utilizada para gerar diferentes realizações de parâmetros, sendo cada realização subsequentemente executada no modelo determinístico (BEVEN; BILEY, 1992). Assim, os resultados da execução do modelo determinístico podem ser analisados estatisticamente. 
Figura 1 - Classificação de modelos de simulação hidrológica

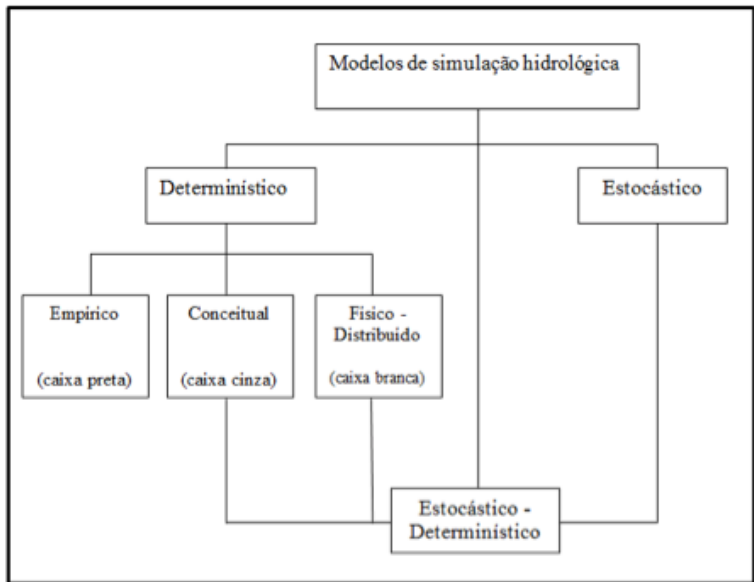

Fonte: Adaptado de Refsgaard (1996).

Por uma perspectiva sistêmica, se pode entender um modelo hidrológico como composto por sete componentes - figura 2 (LIU; GUPTA, 2007): (1) limite do sistema, (2) entradas, (3) condição inicial, (4) parâmetros, (5) estrutura, (6) estados e (7) saídas. Esta concepção é aplicável à maior parte das classes de modelos hidrológicos, à exceção de modelos estatísticos e estocásticos, no qual funções de transferência convertem diretamente séries de entradas em séries de saída (ex. precipitação-vazão ou vazão-vazão).

$$
\text { As entradas (u) e saídas (y) são }
$$
fluxos de massa e energia que respectivamente fluem para dentro e para fora dos limites do sistema (Figura 2). Os estados ( $x$ ) são quantidades de massa e/ou energia que variam temporalmente dentro dos limites do sistema. Ao iniciar a simulação, o estado inicial $\left(\mathrm{x}_{0}\right)$ é representado pela quantidade de massa e/ou energia armazenada no sistema. Os parâmetros $(\theta)$ são propriedades características do sistema, na maioria dos casos são considerados invariantes no tempo (permanecem constantes durante o tempo de simulação). Como uma extensão desta formulação pode-se permitir que as características do sistema (parâmetros) também variem ao longo do tempo em respostas a forçantes externos, como uma alteração no uso da terra.

Figura 2 - Componentes de um modelo de simulação hidrológica

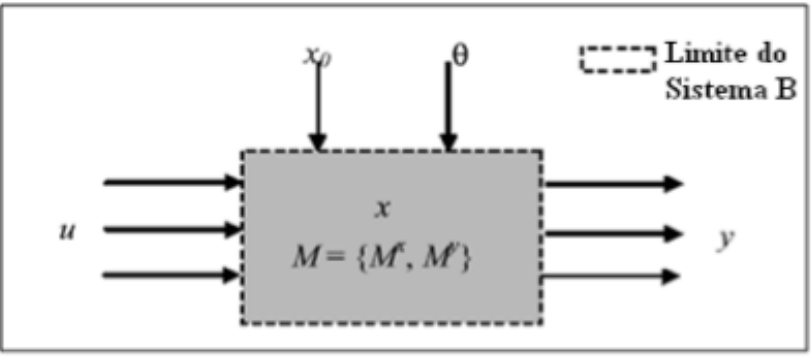

Fonte: Adaptado de Liu e Gupta (2007).

A escolha apropriada do modelo está relacionada com os objetivos a serem alcançados. Para a simulação chuva-vazão de uma bacia em Oklahoma (EUA), por exemplo, Butts et al. (2004) constataram que modelos conceituais, comparativamente aos modelos distribuídos mais complexos, simularam ligeiramente melhor o hidrograma de vazões durante o período de calibração. Neste caso, se aplica o princípio da parcimônia, ou seja, o modelo mais apropriado é aquele que atende o objetivo proposto de forma mais simples e conveniente (GRAYSON; BLÖSCHL, 2002). Já modelos empíricos apresentam menor flexibilidade parar situações fora do contexto de calibração (GOURLEY; VIEUX, 2006), ou seja, podem apresentar desempenho bastante inferior em situação de mudança do padrão de precipitação ou alteração das características superficiais da bacia.

Mas caso o objetivo seja a estimativa de algum componente hidrológico interno da bacia (ex. teor de água no solo), ou a interação entre diferentes componentes do ciclo hidrológico terrestre (ex. relação entre a evapotranspiração real e o teor de água na 
zona radicular), a ferramenta mais apropriada é um modelo hidrológico distribuído. A capacidade de discriminação espacial dos modelos distribuídos também pode ser utilizada para alterar parâmetros espaciais (ex. ligados à cobertura superficial) e avaliar as mudanças na resposta hidrológica da bacia (REFSGAARD, 1996).

\section{Incerteza e assimilação de dados}

Modelos hidrológicos, como representações simplificadas da realidade, estão sujeitos a incertezas. A questão da incerteza em modelagem hidrológica está ligada aos sete componentes citados por Liu e Gupta (2007). Por uma questão de complexidade de representação, conceitual e espacial, modelos distribuídos apresentam maior incerteza comparativamente aos modelos conceituais. As principais diferenças são: (1) complexidade da estrutura do sistema, abrangendo um maior número de processos; (2) necessidade de especificação de um maior número de parâmetros, como, por exemplo, associados aos diferentes tipos de uso da terra, classes de solo e camadas geológicas; (3) representação da heterogeneidade espacial de parâmetros, variáveis de entrada, estados e condições iniciais.

Grayson e Blöschl (2000) defendem a utilização de observações hidrológicas espacializadas para a diminuição de incerteza em modelos distribuídos, chamadas de "padrões espaciais". De acordo com os autores, a utilização de padrões permitem um melhor entendimento dos processos hidrológicos, bem como da quantificação de como estes processos afetam e são afetados pela variabilidade espacial da superfície em diferentes escalas.

A importância de padrões espaciais pode ser atestada pelo crescente número de técnicas disponíveis para a identificação, a quantificação e a redução de incerteza em modelos distribuídos (BEVEN; BILEY, 1992; MADSEN, 2003; VIEUX, 2004; VRUGT et al., 2004; WALKER; HOUSER, 2005). Grayson et al. (2002) destacam a possibilidade de utilização de três tipos de padrões espaciais em modelagem hidrológica:

(1) Pontual. Nesse caso, quando há suficiente número de pontos amostrais se pode reproduzir o padrão espacial de alguma variável / parâmetro hidrológico, sendo possível também analisar a estrutura espacial por meio de técnicas como a variografia.

(2) Binário. São variáveis representadas espacialmente em relação a sua ocorrência ou não, como áreas com cobertura de neve ou inundadas, mas sem representação de valores absolutos.

(3) Correlacionado. A variável apresenta algum grau de correlação com o padrão de interesse, sendo mais fáceis de obtenção em uma forma espacialmente distribuída. Exemplos fornecidos por Grayson et al. (2002) incluem as equações de pedo-transferência (textura do solo para inferir propriedades hidráulicas) e dados de sensoriamento remoto (no qual a emissão e a reflectância da radiação em diferentes comprimentos de onda são correlacionados com feições de interesse, como a cobertura vegetal, a temperatura superficial e a umidade do solo).

Particularmente a utilização de sensoriamento remoto representa uma 
alternativa interessante na diminuição de incerteza de modelos hidrológicos. A aquisição de dados de sensoriamento remoto é por natureza espacial, permitindo que importantes padrões de importância hidrológica sejam correlacionados com a resposta espectral dos alvos em diferentes comprimentos de onda eletromagnética. Acredita-se que apesar das incertezas associadas às estimativas dos modelos distribuídos e de sensoriamento remoto, a possibilidade de combiná-los resulta em significativo benefício mútuo (REFSGAARD, 2000).

A utilização de sensoriamento remoto em hidrologia ganhou impulso na última década, principalmente devido à diversificação de sensores orbitais voltados ao monitoramento de mudanças hidrológicas e climatológicas globais. As aplicações são diversas, incluindo a estimativa de parâmetros superficiais (ex. índice de área foliar, rugosidade superficial, altura dossel etc.), estimativas hidrológicas como precipitação, evapotranspiração, teor de água no solo, total de água armazenada na superfície, nível de rios e reservatórios, vazões e qualidade da água; estimativas auxiliares como temperatura superficial, radiação incidente, vapor de água na atmosfera, entre outros (SCHULTZ; ENGMAN, 2000; LIANG, 2004; LAKSHMI, 2005; CAZENAVE; SAVENIJA, 2008).

O uso do sensoriamento remoto para a diminuição da incerteza de modelos hidrológicos pode se dar de diferentes formas: (1) dados de entrada (ex. precipitação), particularmente importante em áreas com baixa densidade de estações pluviométricas; (2) parametrização de modelos (ex. mapa de índice de área foliar, no modelo associado com a capacidade de água interceptada); (3) atualização de estados (ex. evapotranspiração), no qual a estimativa remota é utilizada para corrigir os estados do modelo (variável interna do sistema) durante a execução da simulação; (4) calibração de parâmetros (ex. evapotranspiração), no qual as estimativas remotas são utilizadas para inferir os valores de parâmetros do modelo hidrológico, principalmente ligados a diferentes coberturas vegetais e solos; (5) validação (ex. áreas alagadas), no qual a observação de sensoriamento remoto é utilizada para verificar a qualidade das saídas do modelo hidrológico.

\section{Evapotranspiração: uma variável-chave}

A disponibilidade hídrica no contexto da bacia hidrográfica sofre influência de diferentes fatores antrópicos, incluindo a dinâmica espacial-temporal do uso da terra, os diferentes usos da água, bem como da presença de infraestruturas hidráulicas, incluindo barragens, diques e canais (ARNELL, 2002). O uso da terra influencia diretamente o balanço hídrico, alterando o tempo e a magnitude da evapotranspiração e, por conseguinte, a quantidade de água no solo e a recarga do aquífero (MAO; CHERKAUNER, 2009).

O termo evapotranspiração abrange todos os processos no qual a água liquida e/ou sólida é transformada em vapor de água na superfície terrestre, incluindo a evaporação da água no dossel das plantas, corpos d'água, solos e a transpiração das plantas (DINGMAN, 2002). 
A evapotranspiração é a maior componente do ciclo hidrológico na sua fase terrestre - em contato com a superfície (FALKENMARK; ROCKSTRÖM, 2005), sendo influenciada pela micrometeorologia, pelo teor de água no solo e aspectos fisionômicos/fisiológicos da vegetação (ALLEN et al., 1998). Essa quantidade de variáveis torna a estimativa da evapotranspiração complexa, já que a evapotranspiração sofre influência de fatores naturais e antrópicos, ligados ao uso da terra e da água (irrigação).

\section{Estimativa da evapotranspiração real por sensoriamento remoto}

O ponto focal para as estimativas de evapotranspiração real utilizando modelos hidrológicos é a consideração do balanço hídrico no solo, pois o estresse hídrico provoca valores de evapotranspiração abaixo das taxas potenciais. Em sensoriamento remoto, diferentes estratégias podem ser utilizadas para se obter estimativas de evapotranspiração real, incluindo métodos empíricos até físicos (GOWDA et al., 2008; KALMAN et al., 2008). Os métodos físicos são baseados no balanço de energia (BASTIAANSSEN et al., 1998a; SU, 2002; ALLEN; TASUMI; TREZZA, 2007), com possibilidade de separação entre a evaporação e a transpiração (NORMAN et al., 1995).

O fluxo de calor latente, correspondente à energia consumida pelo processo evapotranspirativo, é obtido pela equação de balanço de energia uma vez conhecidos o fluxo de calor sensível, o fluxo de calor no solo e o saldo de radiação:

$$
L E=R_{n}-G-H
$$

em que LE = fluxo de calor latente $\left(\mathrm{W} \mathrm{m}^{-2}\right)$; $\mathrm{R}_{\mathrm{n}}=$ saldo de radiação $\left(\mathrm{W} \mathrm{m}^{-2}\right) ; \mathrm{G}=$ fluxo de calor no solo $\left(\mathrm{W} \mathrm{m} \mathrm{m}^{-2}\right) ; \mathrm{H}=$ fluxo de calor sensível $\left(\mathrm{W} \mathrm{m}^{-2}\right)$.

Os algoritmos Tseb - Two-Source Energy Balance (NORMAN et al., 1995), Sebal - Surface Energy Balance Algorithm for Land (BASTIAANSSEN et al., 1998a; 1998b), Metric

- Mapping EvapoTranspiration at high Resolution with Internalized Calibration (ALLEN; TASUMI; TREZZA, 2007; ALLEN et al., 2007) e Sebs - Surface Energy Balance System (SU, 2002) utilizam o balanço de energia para estimar a evapotranspiração real por sensoriamento remoto, no qual o fluxo de calor latente é a única variável não estimada, mas pode ser obtida pela aplicação da equação de balanço de energia.

O saldo de radiação na superfície (W $\mathrm{m}^{-2}$ ), que representa a energia radiante disponível para os processos biofísicos (CAMPBELL; NORMAN, 1998), é calculado pela diferença entre os fluxos radiantes refletidos ou emitidos pela superfície e os fluxos radiantes incidentes na mesma superfície:

$$
R_{n}=R_{S \downarrow}-a R_{S \downarrow}+R_{L \downarrow}-R_{L \uparrow}-\left(1-\varepsilon_{0}\right) R_{L \downarrow}
$$

em que $R_{S_{\downarrow}}=$ radiação em ondas curtas incidente $\left(\mathrm{W} \quad \mathrm{m}^{-2}\right) ; \quad \mathrm{a}=$ albedo (adimensional); $\mathrm{R}_{\mathrm{L} \downarrow}=$ radiação em ondas longas incidente $\left(\mathrm{W} \mathrm{m} \mathrm{m}^{-2}\right) ; \mathrm{R}_{\mathrm{L} \uparrow}=$ radiação emitida em ondas longas; $\varepsilon_{0}=$ emissividade da superfície (adimensional).

Em geral, os algoritmos para obtenção da evapotranspiração apresentam pequenas variações no cálculo do saldo de radiação. As radiações incidentes de ondas curtas e longas podem ser obtidas por simples esquemas de parametrização, considerando as condições posicionais e 
topográficas, como dia do ano, hora do dia, latitude, longitude, aspecto e declividade do terreno etc. (ALLEN; TASUMI; TREZZA, 2007); ou estimadas a partir de dados de imagens de satélites com auxílio de modelos de transferência radiativa (WANG; LIANG, 2009); ou medidas diretamente no terreno (SU, 2002).

O albedo, a emissividade e a radiação emitida de ondas longas são estimados por meio de técnicas de sensoriamento remoto. Os valores de albedo geralmente são estimados por meio da combinação linear da reflectância bi-direcional de diferentes bandas espectrais situadas entre o visível e o infravermelho próximo (TASUMI et al., 2008; WUBET, 2003). A emissividade da superfície pode ser estimada por meio de equações empíricas utilizando índices de vegetação (BASTIAANSSEN et al.,1998a; SOBRINO et al., 2001; ALLEN; TASUMI; TREZZA, 2007), ou associadas com mapas de uso da terra (SNYDER et al., 1998), ou estimadas de forma iterativa por meio de múltiplas bandas termais (GILLESPIE et al., 1998). Para o sensor MODIS (produto MOD11), a emissividade é estimada pelo "método da classificação", no qual o pixel da imagem é classificado de acordo com 14 classes de uso da terra pré-definidas e associado com valores de emissividade tabelados (SNYDER et al., 1998). No algoritmo Metric, Allen, Tasumi e Trezza (2007) usam uma simples equação empírica, que correlaciona linearmente a emissividade com o índice de área foliar:

$$
\begin{aligned}
& \varepsilon=0,95+0,01 \mathrm{IAF}, \text { para } \mathrm{IAF} \leq 3 \\
& \varepsilon=0,98, \text { para IAF } \geq 3
\end{aligned}
$$

em que $\varepsilon$ é a emissividade da superfície e IAF, o índice de área foliar.
Essa equação empírica foi obtida a partir de dados globais de emissividade termal de solos e vegetação da biblioteca espectral da Universidade de Santa Bárbara EUA (UCSB Emissivity Library). A radiação emitida em ondas longas é calculada usando a equação de Stefan-Boltzmann.

O fluxo de calor do solo depende da condutividade termal e do gradiente vertical de temperatura do solo, representando normalmente cerca de 5 a $20 \%$ do saldo de radiação (KALMA et al., 2008). Como não é possível a medida remota do fluxo de calor no solo, a fração $G / R_{n}$ pode ser assumida como uma constante ou calculada empiricamente através de um índice de vegetação, da temperatura da superfície e do albedo da superfície. $\mathrm{Na}$ formulação de Bastiaanssen et al. (1998a), a fração $G / R_{n}$ aumenta com $\mathrm{o}$ albedo (inversamente correlacionado com a umidade do solo) e diminui em sentido oposto à cobertura vegetal, representando a atenuação da radiação eletromagnética através do dossel das plantas.

Para a estimativa do fluxo de calor sensível é necessário o uso de um sensor termal, que mede a temperatura radiométrica da superfície. A temperatura radiométrica é derivada do balanço de radiação de uma superfície, no qual a emissão da radiação em ondas longas em uma superfície está correlacionada com sua temperatura superficial (NORMAN; BECKER, 1995). O algoritmo Sebal usa o gradiente de temperatura (dT) em detrimento da temperatura radiométrica. De forma aproximada, dT corresponde à diferença de temperatura entre a superfície e a atmosfera adjacente, promovendo o fluxo de calor sensível. 
O gradiente de temperatura próximo à superfície (dT) é obtido por meio de uma indexação linear com a temperatura superficial $\left(T_{S}\right)$ :

$$
d T=a+b T_{s}
$$

Os coeficientes "a" e "b" são obtidos para cada imagem tomando-se como base dois pixels em situações extremas em termos da partição de energia, no qual dT pode ser estimado: "pixel frio" e "pixel quente". O "pixel frio" é geralmente selecionado em uma superfície líquida, no qual se assume que o fluxo de calor sensível pode ser nulo $(\mathrm{H}=0 \mathrm{e}$ $\mathrm{dT}=0$ ) e, portanto, toda a energia disponível $\left(R_{n}-G\right)$ seria utilizada no processo evaporativo. Já o "pixel quente" é selecionado em uma superfície na qual não ocorreria evaporação $\left(H=R_{n}-G\right)$. A partir desse ponto, se conhece dois pares da temperatura radiométrica e do gradiente de temperatura na imagem de satélite ( $\mathrm{dT}_{\text {quente }} \mathrm{e}$ $\mathrm{T}_{\text {quente}} ; \mathrm{dT}_{\text {frio }}$ e $\mathrm{T}_{\text {frio }}$ ). Uma regressão linear simples é utilizada para se obter o intercepto e o coeficiente angular da regressão entre o gradiente de temperatura e a temperatura superficial ("a" e "b" da equação anterior). A equação anterior é então aplicada a todos os pixels da imagem, resultando no gradiente de temperatura e, pela aplicação da equação posterior, em uma primeira estimativa do fluxo de calor sensível.

$$
\mathrm{H}=\rho_{\mathrm{ar}} \mathrm{C}_{\mathrm{p}} \frac{\mathrm{dT}}{\mathrm{r}_{\mathrm{ah}}}
$$

em que $\rho_{a r}=$ densidade do $\operatorname{ar}\left(\mathrm{kg} \mathrm{m}^{-3}\right) ; C_{p}=$ calor específico do ar em pressão constante ( $\mathrm{kg}^{-1} \mathrm{~K}^{-1}$ ) e $\mathrm{r}_{\mathrm{ah}}=$ resistência aerodinâmica ao fluxo de calor sensível $\left(\mathrm{s} \mathrm{m}^{-1}\right), \mathrm{dT}=$ gradiente de temperatura próximo a superfície (K), de forma aproximada, dT corresponde a diferença de temperatura entre a superfície e a atmosfera adjacente, promovendo o fluxo de calor sensível.

\section{Validação de estimativas remotas de evapotranspiração}

Bastiaanssen et al.

apresentaram uma série de 18 experimentos de validação do Sebal, abrangendo diferentes condições de umidade do solo e coberturas vegetais. A exatidão média na escala diária encontrada foi de $85 \%$, aumentando para 96\% em escala sazonal devido à anulação de erros aleatórios. Allen et al. (2007) realizaram a validação do Metric utilizando dois lisímetros instalados em Idaho-EUA. Para a estação de crescimento das culturas agrícolas (4 meses), os erros relativos na escala sazonal foram de $1 \%$ e $4 \%$, sendo os desvios-padrão dos erros no momento de passagem do satélite respectivamente de 13 e 20\%. Kalman et al.(2008) sintetizaram uma série de 30 estudos de validação para diferentes algoritmos empíricos e físicos para calcular evapotranspiração. Para o fluxo de calor latente, o erro médio quadrático

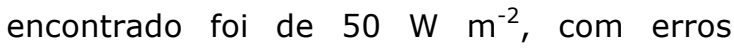
relativos variando entre 15 e $30 \%$ no momento de aquisição da imagem. Desses estudos, seis são relativos ao TSEB, apresentando erro médio relativo de $19 \%$ no momento da passagem do satélite.

\section{Utilização de estimavas remotas de evapotranspiração em modelos hidrológicos}

Assimilação de dados refere-se ao emprego de observações visando à diminuição de incerteza em modelos. A assimilação de dados de sensoriamento 
remoto tem sido empregada com sucesso há décadas na meteorologia e na oceanografia, tendo sido usada apenas recentemente na hidrologia, principalmente na atualização de estado do teor de água no solo, mas também com exemplos envolvendo observações de temperatura superficial, neve, armazenamento de água superficial e vazão (WALKER; HOUSER, 2005).

Estudos de assimilação de evapotranspiração real são ainda mais recentes, sendo baseados na calibração de parâmetros ligados à evapotranspiração simulada pelo modelo hidrológico (INES; DROOGERS, 2002a; JHORAR et al., 2002; IMMERZEEL; DROOGERS, 2008), atualização de estados, ou seja, a correção dos valores de evapotranspiração simulados pelo modelo hidrológico (SCHUURMANS et al., 2003; PIPUNIC et al., 2008; QIN et al., 2008) ou restrição do espaço de parâmetros de modelos estocásticos (WINSEMIUS et al., 2008). Baseados em diferentes critérios de validação, todos os estudos acima destacam o impacto positivo da assimilação de evapotranspiração.

A calibração de parâmetros do modelo hidrológico é um procedimento frequentemente utilizado. Conforme destacado, parâmetros são características do sistema, relacionados, por exemplo, ao tipo de vegetação ou solo. Em uma abordagem usual, os parâmetros do modelo hidrológico são definidos previamente, associados a um mapa de uso da terra e a evapotranspiração é uma variável de saída do modelo hidrológico. Mas existe uma incerteza muito grande em relação aos valores ou aos limites de valores de parâmetros representativos de cada classe de uso da terra. Uma alternativa é a inversão de modelos hidrológicos (calibração), no qual o conjunto de parâmetros ótimo é selecionado, por meio da aplicação de alguma técnica de otimização, visando minimizar as diferenças entre as estimativas de evapotranspiração do modelo e as obtidas por sensoriamento remoto. Neste caso, o procedimento de calibração permite a seleção do conjunto de parâmetros do modelo hidrológico que produza uma estimativa de evapotranspiração semelhante à observada remotamente.

\section{Limitações dos procedimentos de assimilação de estimavas remotas de evapotranspiração em modelos hidrológicos}

\section{Ausência da abordagem probabilística}

Beven e Biley (1992) destacam que no contexto da modelagem hidrológica existem diferentes modelos e conjuntos de parâmetros, fisicamente embasados (não violam os princípios físicos), capazes de simular de forma consistente as observações coletadas em campo (ex. hidrograma). Este fenômeno recebe a denominação de equifinalidade. Este problema permanece mesmo se houvesse um modelo "verdadeiro", pois seria necessário a identificação do conjunto de parâmetros apropriado para uma área específica usando limitadas observações. Frequentemente ocorre que diferentes conjuntos de parâmetros são consistentes com os dados observados.

Para lidar com o problema da equifinalidade, Beven e Biley (1992) propõem o método Generalized Likelihood Uncertainty Estimation Methodology - Glue. Basicamente a metodologia Glue prevê e execução do modelo com diferentes realizações de parâmetros, sendo subsequentemente 
selecionados apenas os conjuntos de parâmetros aceitáveis (behavioral), ou seja, que estão em maior concordância com observações coletadas na bacia hidrográfica simulada. As simulações derivadas de todos os conjuntos de parâmetros aceitáveis são ponderadas e utilizadas conjuntamente para produzir uma estimativa de incerteza.

Nesta metodologia não há otimização, pois não existe um único conjunto ótimo de parâmetros em função das incertezas inerentes a modelagem hidrológica.

Apesar das incertezas ligadas a parametrização da evapotranspiração em modelos hidrológicos e da estimativa usando a equação de balanço de energia por sensoriamento remoto, o único trabalho identificado a tratar explicitamente da questão da equifinalidade foi o de Winsemius et al.(2008). Neste trabalho, um modelo conceitual semidistribuído foi utilizado juntamente com simulações de Monte Carlo. Inicialmente o modelo foi executado com diferentes realizações de Monte Carlo, sendo a seguir selecionadas as realizações com menores discrepâncias entre a evapotranspiração simulada e observada (Sebal). Foi constatada uma maior restrição do espaço de parâmetros ligados diretamente a evapotranspiração, resultando em uma interpretação mais clara do comportamento da evapotranspiração para diferentes coberturas de uso da terra.

\section{Desagregação representatividade estimativas evapotranspiração}

$\begin{array}{cr}\text { espacial } & \text { e } \\ \text { temporal } & \text { das } \\ \text { remotas } & \text { de }\end{array}$

A qualidade do procedimento de calibração depende intrinsecamente da qualidade das estimativas obtidas por sensoriamento remoto. Na atual geração de satélites as opções de sensores termais necessários para o cálculo da evapotranspiração real são restritas em relação à frequência de imageamento e à resolução espacial. As opções atuais são sensores termais de baixa resolução espacial e alta resolução temporal (MODIS, AVHRR), já que os sensores termais de média resolução espacial e baixa resolução temporal (ETM, ASTER) encontram-se inativos.

Idealmente para os procedimentos de calibração seria interessante ter estimativas remotas de evapotranspiração com frequência temporal diária e com média resolução espacial (< $100 \mathrm{~m}$ ). A resolução espacial deve ser suficientemente boa para se estabelecer uma relação biunívoca entre a medida de evapotranspiração remota e um determinado polígono de uso da terra representado no modelo hidrológico. A alta frequência temporal é necessária para se capturar a dinâmica da evapotranspiração ao longo do tempo, já que essa sofre alteração em função da quantidade de água precipitada ou aplicada por irrigação sobre o solo.

A questão da escala nas estimativas de evapotranspiração tem sido alvo de pesquisa, incluindo estudos que artificialmente degradam a resolução espacial de dados radiométricos (SU et al., 1999; KUSTAS et al., 2003; LI et al., 2008; HONG 2009), ou que fizeram a comparação direta entre estimativas obtidas de diferentes satélites (MORAN et al., 1997; MCCABE; WOOD, 2006).

Moran et al. (1997) constataram que a agregação espacial de uma série de variáveis obtidas por sensoriamento remoto é 
linear em diferentes escalas, incluindo a reflectância, o albedo e diferentes índices de vegetação. Isto significa que a estimativa em baixa resolução espacial é equivalente à média aritmética das estimativas realizadas em melhores resoluções espaciais. Para a temperatura da superfície também se pode assumir linearidade para a maioria dos intervalos de medida. Porém, constatou-se que a agregação dos componentes do balanço de energia é mais complexa, resultando em erros significativos nas diferentes escalas espaciais.

Su et al. (1999) analisaram duas situações distintas de agregação de estimativas de evapotranspiração usando o Sebal: (1) inicialmente, o Sebal foi executado na resolução de 18,5 metros e posteriormente as estimativas de evapotranspiração foram degradadas em sete resoluções (37, 50, 74, 148, 296, 592 e 1184 metros); (2) as variáveis-parâmetro obtidas pelo satélite foram degradadas nas mesmas sete resoluções e na sequência o Sebal foi executado. A comparação das estimativas degradadas com a superfície de evapotranspiração na escala original (18,5 metros) evidenciou um erro quase desprezível usando a proposição (1), enquanto que a utilização da proposição (2) resultou em significativos erros, variando de 30 a $60 \%$ do fluxo de calor latente. Essa conclusão é semelhante à obtida por Hong (2009), ou seja, as estimativas do fluxo de calor latente não são lineares em diferentes resoluções espaciais usando o Sebal.

$\mathrm{Li}$ et al. (2008) destacam que o impacto de utilização de diferentes resoluções de entrada no TSEB depende intrinsecamente da homogeneidade do terreno e do espaçamento entre diferentes usos da terra.
Para o caso analisado em Charleston (Arizona-EUA), a diferença do fluxo de calor latente entre as resoluções de 50 e 500 metros chegou a $200 \mathrm{~W} \mathrm{~m} \mathrm{~m}^{-2}$. Kustas et al. (2004), também utilizando o TSEB, perceberam diferenças significativas no histograma de imagens de evapotranspiração nas resoluções de 60, 120, 240 e 960 metros. Apenas nas resoluções de 60 e 120 metros se pode visualizar dois picos no histograma da evapotranspiração, correspondentes às plantações de milho e soja.

Inúmeras técnicas têm sido propostas para a construção de séries temporais de evapotranspiração a partir do uso de satélites. Em geral, se pode agrupar essas técnicas em dois grupos: 1) técnicas de interpolação temporal das estimativas de evapotranspiração feitas a partir de sensores termais de média resolução espacial e baixa frequência temporal (BASTIAANSSEN, 1998a; ALLEN; TASUMI; TREZZA, 2007); 2) técnicas de desagregação espacial das estimativas de evapotranspiração feitas a partir de sensores termais de baixa resolução espacial e alta frequência temporal (CHEMIN; ALEXANDRIDIS, 2004; KUSTAS et al., 2003; VAZIFEDOUST, 2007).

Em função da inativação dos sensores TM e ASTER, as técnicas construção de séries temporais de evapotranspiração estão atualmente limitadas ao segundo grupo. Nos trabalhos consultados, apenas Immerzeel e Droogers (2008) utilizam algum procedimento de desagregação das estimativas de evapotranspiração anteriormente ao procedimento de assimilação. 
Immerzeel e Droogers (2008) usaram 16 imagens MODIS, distribuídas em um período de 8 meses, para calcular evapotranspiração usando o algoritmo Sebal. Posteriormente, as estimativas remotas de evapotranspiração foram agrupadas em valores mensais por sub-bacia. O modelo utilizado foi o SWAT (Soil and Water Assessment Tool), sendo cinco parâmetros calibrados por meio do algoritmo GML (Gauss-Marquardt-Levenberg) usando as estimativas remotas de evapotranspiração como referência. A validação foi feita tomando-se como base os dados históricos de vazão da bacia hidrográfica. O modelo calibrado com dados remotos de evapotranspiração apresentou maior aproximação com os dados monitorados de vazões. Apesar da construção de uma série temporal diária, os valores de evapotranspiração foram durante a aplicação do procedimento de assimilação agrupados na escala mensal por sub-bacia.

O aspecto da desagregação torna-se fundamental ao se trabalhar em áreas com cobertura da superfície heterogênea, pois se deve garantir a correspondência direta dos parâmetros de vegetação e solo do modelo representados no modelo (célula do modelo distribuído) e a observação do satélite (pixel), pois a estimativa de evapotranspiração não é linear em diferentes escalas espaciais (MORAN et al., 1997; KUSTAS et al., 2004; MCCABE; WOOD, 2006; LI et al., 2008). Esse aspecto não tem sido considerado nos procedimentos de assimilação da evapotranspiração.
Validação limitada dos procedimento de assimilação

A avaliação das estimativas distribuídas de evapotranspiração, obtidas remotamente e por modelagem, é complexa, principalmente pela ausência de uma "superfície real de evapotranspiração", já que em geral dados de evapotranspiração são monitorados pontualmente por meio de experimentos agrometeorológicos (TEIXEIRA et al., 2009).

A assimilação da evapotranspiração obtida remotamente em modelos distribuídos permite a simulação integrada do ciclo hidrológico terrestre, possibilitando simular a série temporal de vazões observadas em um rio, ou dados pontuais de umidade do solo e da altura do lençol freático, que também podem ser comparados com dados observados em campo, corroborando com a validação das estimativas remotas.

As estratégias de validação encontradas nas referências foram: (1) a comparação relativa entre superfícies de evapotranspiração obtidas remotamente e calculadas por modelos hidrológicos sem e com assimilação de dados (SCHUURMANS et al., 2003; PIPUNIC et al., 2008; QIN et al., 2008; PAN et al., 2008); (2) restrição do espaço de parâmetros (WINSEMIUS et al., 2008); (3) comparação dos parâmetros do modelo após assimilação, utilizando valores de referência obtidos in situ ou na bibliografia (INES; DROOGERS,2002a); (4) impacto sobre o balanço hídrico, como a melhoria do hidrograma simulado (IMMERZEEL; DROOGERS, 2008). 
Em nenhuma das referências citadas foram utilizados experimentos agrometeorológicos nos procedimentos de validação, incluindo o monitoramento da evapotranspiração real e/ou do teor de água no solo em diferentes pontos da bacia hidrográfica. Teixeira et al. (2009) utilizam uma série de experimentos agrometeorológicos ("Razão de Bowen" e "Fluxos Turbulentos") para calibrar e validar estimativas remotas de evapotranspiração obtidas pela aplicação do Sebal no Sub-Médio São Francisco. Bastiaanssen et al. (2005) e Allen et al. (2007) também apresentam uma série de estudos de validação dos algoritmos Sebal e Metric por meio de lisímetro.

Esses procedimentos de validação, amplamente utilizados em estimativas remotas, poderiam ser utilizados para analisar o impacto da assimilação da evapotranspiração em modelos hidrológicos distribuídos, permitindo uma avaliação direta da qualidade do dado simulado em relação ao coletado em campo.

\section{Conclusões}

Modelos hidrológicos são ferramentas úteis para identificar e quantificar o padrão espacial da água em sua fase terrestre, bem como para analisar a influência do uso da terra e da água sobre o ciclo hidrológico. Em particular, a evapotranspiração é uma variável-chave, que liga fatores antrópicos e naturais, influenciando a quantidade de água disponível no solo e que vai recarregar o aquífero.
Mas existe uma incerteza muito grande na simulação da evapotranspiração utilizando modelos hidrológicos, principalmente ligada à definição de parâmetros característicos de cada classe de uso da terra. Por outro lado, técnicas para a estimativa de evapotranspiração por sensoriamento remoto atingiram um relativo grau de maturidade. Essas estimativas remotas podem ser utilizadas de diferentes formas para diminuir a incerteza de modelos hidrológicos.

A integração das técnicas de modelagem hidrológica e sensoriamento remoto ocasiona beneficio mútuo em relação à representação espacial da evapotranspiração, ou seja, do ponto de vista da modelagem hidrológica há diminuição da incerteza, enquanto que do ponto de vista do sensoriamento remoto a integração com todo o ciclo hidrológico terrestre permite uma avaliação mais completa da qualidade da superfície de evapotranspiração estimada remotamente.

Para o avanço no tema, destacam-se os seguintes pontos: (1) uso de técnicas de desagregação espacial de estimativas remotas de evapotranspiração antes do procedimento de assimilação; consideração de incerteza na definição dos parâmetros do modelo; (3) coleta de dados hidrológicos em diferentes pontos da bacia hidrográfica, permitindo uma avaliação mais completa das simulações do modelo hidrológico sem e com a assimilação de estimativas de evapotranspiração obtidas remotamente. 


\section{Referências bibliográficas}

ALLEN, R.; PEREIRA, L. S.; RAES, D.; SMITH, $M$. Crop evapotranspiration: guidelines for computing crop water requirements. Roma: 1998. (FAO Irrigation and Drainage Paper, v. 56).

ALLEN, R. G.; TASUMI, M.; TREZZA, R. Satellite-based energy balance for mapping evapotranspiration with internalized calibration (METRIC) model. Journal of Irrigation and Drainage Engineering, v. 133, n. 4, p. 380-394, 2007.

ALLEN, R. G.; TASUMI, M.; MORSE, A.; TREZZA, R.; WRIGHT, J. L.; BASTIAANSSEN, W.; KRAMBER, W.; LORITE, I.; ROBISON, C. W. Satellitebased energy balance for mapping evapotranspiration with internalized calibration (METRIC) - applications. Journal of Irrigation and Drainage Engineering, v. 133, n. 4), p. 395406, 2007.

\section{ARNELL, N. Hydrology \& Global} Environmental Change. Londres: Pretice Hall, 2002.

BASTIAANSSEN, W.; NOORDMAN, E. J. M.; PELGRUM, H.; DAVIDS, G.; THORESON, B. P.; ALLEN, R. G. SEBAL model with remotely sensed data to improve water-resources management under actual field conditions. Journal of Irrigation and Drainage Engineering, v. 131, n. 1 , p. 85-93, 2005.

BASTIAANSSEN, W. G. M.; MENENTI, M.; FEDDES, R. A.; HOLTSLAG, A. A. M. A remote sensing surface energy balance algorithm for land (SEBAL): 1. Formulation. Journal of Hydrology, v. 213, n. 1-4, p. 198212, 1998a.

BASTIAANSSEN, W. G. M.; MENENTI, M.; FEDDES, R. A.; HOLTSLAG, A. A. M. A remote sensing surface energy balance algorithm for land (SEBAL): 2. Validation. Journal of Hydrology, v. 213, n. $1-4$, p. 213-229, 1998 b.

BEVEN, K.; BILEY, A. M. The future of distributed models: model calibration and uncertainty prediction.

Hydrological Processes, v. 6, p. 279-298, 1992.
BUTTS, M. B. ; PAYNEA, J. T.; KRISTENSENB, M.; MADSEN, H. An evaluation of the impact of model structure on hydrological modeling uncertainty for streamflow simulation. Journal of Hydrology, v. 298, p. 241-250, 2004.

CAMPBELL, G. S.; NORMAN, J. M. An Intoduction to Environmental Biophysiscs. Nova York: Springer, 1998.

CARPENTER, T. M.; GEORGAKAKOS, K. P. Continuous Streamflow Simulation with the HRCDHM Distributed Hydrologic Model. Journal of Hydrology, v. 298, p. 61-79, 2004.

CAZENAVE, A.; SAVEniJA, H. Preface to the special issue of hydrology from space. Survey Geophysics, v. 29, p. 241245, 2008.

CHEMIN, Y.; ALEXANDRIDIS, T. Improving spatial resolution of ET seasonal for irrigated rice in Zhanghe, China. Asian Journal of Geoinformatics, v. 5, n. 1, p. 3-11, 2004.

CHORLEY, R. J. Introduction to Geographical Hydrology. Londres, Methuen, 1971.

DHI. DANISH HYDRAULIC INSTITUTE. MIKESHE user manual. Estocolmo: DHI, 2008.

DIGMAN, S. L. Physical Hydrology. 2. ed. Long Grove: Waveland Press, 2002.

FALKENMARK, M.; ROCKSTRÖM, J. Balancing water for humans and nature. 2. ed. Londres: Earthscan, 2005.

GILLESPIE, A.; ROKUGAWA, S., MATSUNAGA, T., COTHERN, J. S., HOOK, S.; KAHLE, A. B. A Temperature and Emissivity Separation Algorithm for Advanced Spaceborne Thermal Emission and Reflection Radiometer (ASTER) Images. Transactions on Geoscience and Remote Sensing, v. 36 , p. $1113-1126,1998$.

GRAYSON, R. B.; BLÖSCHL, G. Spatial patterns in catchment hydrology. Cambridge: Cambridge University Press, 2000. 
GOURLEY, J. J.; VIEUX, B. E. A method for identifying sources of model uncertainty in rainfall-runoff simulations. Journal of Hydrology, v. 327, p. $68-80,2006$

GOWDA, P. H.; CHAVEZ, J. L. ; COLAIZZI, P. D.; EVETT, S. R.; HOWELL, T. A. ; TOLK, J. A. ET mapping for agricultural water management: present status and challenges. Irrigation Science, v. 26, n. 3, p. 223-237, 2008.

HONG, S. Up-scaling of SEBAL derived evapotranspiration maps from LANDSAT $(30 \mathrm{~m})$ to MODIS $(250 \mathrm{~m})$ scale. Journal of Hydrology, v. 370, p. $122-138,2009$.

KNAPP, B. J. Elements of Geographical Hydrology. Londres: Routledge, 1979.

IMMERZEEL, W. W.; DROOGERS, P. Calibration of a distributed hydrological model based on satellite evapotranspiration. Journal of Hydrology, v. 349, p. 411-424, 2008.

INES, A. V. M.; DROOGERS, P. Inverse modeling in estimating soil hydraulic functions: a genetic algorithm approach. Hydrology and Earth System Sciences, v. 6, p. 49.65, 2002.

JHORAR, R. K.; BASTIAANSSEN, W. G. M.; FEDDDES, R. A.; VAN DAM, J. C. Inversely estimating soil hydraulic functions using evapotranspiration fluxes. Journal of Hydrology, v. 258, p. $198-213,2002$.

KALMAN, J. D.; MCVICAR, T. R.; MCCABE. M. $F$. Estimating land surface evaporation: a review of methods using remotely sensed surface temperature data. Surveys in Geophysics, v. 29 , n. $4-5$, p. 421469, 2008.

KUSTAS, W. P.; NORMAN, J. M.; ANDERSON, M. C.; FRENCH, A. N. Estimating subpixel surface temperatures and energy fluxes from the vegetation index-radiometric temperature relationship. Remote Sensing of Environment, v. 85, p. 429-440, 2003.
KUSTAS, W. P.; JACKSONA, T. J.; PRUEGERB, J. H.; MACPHERSONC, J. I.; WOLDE, $M$. Effects of remote sensing pixel resolution on modeled energy flux variability of croplands in Iowa.

Remote Sensing of Environment, v. 92, p. 535-547, 2004.

LAKSHMI, V. Remote sensing and hydrology. In: ASWATHANARAYANA, U. (Org.). Advances in water science methodologies. Londres: Taylor \& Francis, 2005, p. 3-24.

LI, F.; KUSTAS, W. P.; ANDERSON, M. C.; PRUEGER, J. H.; SCOTT, R. L. Effects of remote sensing spatial resolutions on interpreting tower-based flux observations. Remote Sensing of Environment, v. 112, p. 337-349, 2008.

LIANG, S. Quantitative remote sensing of land surface. Nova Jersey: Jown Wiley \& Sons, 2004.

LIU, Y.; GUPTA, H. V. Uncertainty in hydrologic modeling: towards an integrated data assimilation framework. Water Resources Research, v. 43, n. 7, 2007.

MADSEN, $\mathrm{H}$. Parameter estimation in distributed hydrological catchment modelling using automatic calibration with multiple objectives. Advances in Water Resources, v. 26, p. 205216, 2003.

MAO, D.; CHERKAUER, K. A. Impacts of landuse change on hydrologic responses in Great Lakes region. Journal of Hydrology, v. 374, p. 71-82, 2009.

MACCABE, M. F.; WOOD, E. F. Scale influences on the remote estimation of evapotranspiration using multiple satellite sensors. Remote Sensing of Environment, v. 105, p. 271-285, 2006.

MORAN, M. S.; HUMES, K. S., PINTER JR., P. J. The scaling characteristics of remotely-sensed variables for sparsely-vegetated heterogeneous landscapes. Journal of Hydrology, v. 190, p. 337-362, 1997.

MULLIGAN, M.; WAINWRIGHT, J. Modelling and model building. In: MULLIGAN, M.; WAINWRIGHT, J. (Orgs.). Environmental modelling: finding simplicity in complexity, 
Chichester: John Wiley \& Sons, 2004, p. 5-68.

NORMAN, J. M.; BECKER, F. Terminology in thermal infrared remote sensing of natural surfaces. Agricultural and Forest Meteorology, v. 77, p. 153166, 1995.

NORMAN, J. M.; KUSTAS, W. P.; HUMES, K. S. Source approach for estimating soil and vegetation energy fluxes in observations of directional radiometric surface temperature. Agricultural and Forestry Meteorology, v. 77, p. 263-293, 1995.

PIPUNIC, R. C.; WALKER, J. P.; WESTERN, A. Assimilation of remotely sensed data for improved latent and sensible heat flux prediction: a comparative synthetic study. Remote sensing of Environment, v. 112, p. 1295-1305, 2008.

QIN, C.; JIA, Y.; SU, Z.; ZHOU, Z.; QIU, Y.; SUHUI, $S$. Integrating remote sensing information into a distributed hydrological model for improving water budget predictions in largescale basins through data assimilation. Sensors, v. 8, p. 44414465, 2008.

REFSGAARD, J. C.; ABBOTT, M. B. Distributed hydrological modelling. Dordrecht: Kluwer Academic Publishers, 1996.

REFSGAARD, J. C. Towards a formal approach to calibration and validation of models using spatial data. In: GRAYSON, R. B.; BLÖSCHL, G. (Orgs.). Spatial patterns in catchment hydrology. Cambridge: Cambridge University Press, 2000. p. 329-354.

SCHULTZ, G. A.; ENGMAN, E. T. Remote sensing in hydrology and water management. Nova York: Springer, 2000.

SCHUURMANS, J. M.; TROCH, P. A.; VELDHUIZEN, A. A.; BASTIAANSSEN, W. G. M.; BIERKENS, M. F. P. Assimilation of remotely sensed latent heat flux in a distributed hydrological model. Advances in Water

Resources, v. 26, p. 151-159, 2003.
SNYDER, W. C.; WAN, Z.; ZHANG, Y.; FENG, Y.-Z. Classification-based emissivity for land surface temperature measurement from space.

International Journal of Remote Sensing, v. 19, p. 2753-2774, 1998.

SOBRINO, J. A.; RAISSOUNI, N.; LI, Z.-L. A comparative study of land surface emissivity retrieval from NOAA data. Remote Sensing of Environment, v. 75, p. 256-266, 2001.

SU, Z.; PELGRUM, H.; MENENTI, M. Aggregation effects of surface heterogeneity in land surface processes. Hydrology and Earth System Sciences, v.3, n. 4, p. 549563, 1999.

SU, Z. The surface energy balance system (SEBS) for estimation of turbulent heat fluxes. Hydrology and Earth System Sciences, v.6, n. 1, p. 8599, 2002.

TASUMI, M.; ALLEN, R. G.; TREZZA, R. Atsurface reflectance and albedo from satellite for operational calculation of land surface energy balance. Journal of Hydrologic Engineering, 2008, v. 13, p. 51-63, 2008.

TEIXEIRA, A. H. C.; BASTIAANSSEN, W. G. M.; AHMAD, M. D.; BOS, M. G. Reviewing SEBAL input parameters for assessing evapotranspiration and water productivity for the Low-Middle São Francisco River basin, Brazil Part A: Calibration and validation. Agricultural and Forestry Meteorology, v. 149, p. 462-476, 2009.

TUCCI, C. E. M. Modelos hidrológicos. Porto Alegre: Editora da UFRGS, 1998.

\section{VAZIFEDOUST, M. Development of an} agricultural drought assessment system: integration of agrohydrological modelling, remote sensing and geographical information. Tese (Doutorado em Ciência da Geoinformação e Observação Terrestre) - Wageningen University, Wageningen, 2007.

VIEUX, B. E. Distributed hydrological modeling using GIS. 2. ed. Dordrecht: Kluwer Academic publishers ed, 2004. 
VRUGT, J. A.; DIKS, C. G. H.; GUPTA, H. V.; BOUTEN, W.; VERSTRATEN, J. M. Improved treatment of uncertainty in hydrologic modeling: combining the strengths of global optimization and data assimilation. Water Resources Research, v. 41, p. 1-17, 2005.

WALKER, J. P.; HOUSER, P. R. Hydrologic data assimilation. In: ASWATHANARAYANA, U. (Org.). Advances in water science methodologies. Londres: Taylor \& Francis, p. 25-48, 2005.

WANG, W.; LIANG, S. Estimation of highspatial resolution clear-sky longwave downward and net radiation over land surfaces from MODIS data. Remote
Sensing of Environment, v. 113, p. 745-754, 2009.

WINSEMIUS, H. C.; SAVENIJE, H. H. G.; BASTIAANSSEN, W. G. M.

Constraining model parameters on remotely sensed evaporation: justification for distribution in ungauged basins? Hydrology and Earth System Sciences, v.12, p. 1403-1413, 2008.

WUBET, M. T. Estimation of absolute surface temperature by satellite remote sensing. Dissertação (Mestrado em Ciência da Geoinformação e Observação Terrestre) - ITC, Utrecht, 2003. 